\title{
Blade-Type Reaction Front in Micrometer-sized Germanium Particles during Lithiation
}

\author{
Xinwei Zhou ${ }^{1,2}$, Tianyi Li ${ }^{1}$, Yi Cui ${ }^{1}$, Melissa L. Meyerson ${ }^{3}$, Jason A. Weeks ${ }^{3}$, C. Buddie \\ Mullins $^{3}$, Yang Jin ${ }^{4}$, Hosop Shin ${ }^{1}$, Yuzi Liu ${ }^{2 *}$, and Likun Zhu ${ }^{1 *}$ \\ ${ }^{1}$ Department of Mechanical and Energy Engineering, Indiana University Purdue University \\ Indianapolis, Indianapolis, IN 46202
}

${ }^{2}$ Center for Nanoscale Materials, Argonne National Laboratory, 9700 South Cass Avenue, Argonne, Illinois 60439

${ }^{3}$ Departments of Chemical Engineering and Chemistry, University of Texas at Austin, Austin, TX 78712

${ }^{4}$ Department of Electrical Engineering, Zhengzhou University, Zhengzhou, P. R. China

*Corresponding author: 1ikzhu@iupui.edu (L.Zhu), yuziliu@anl.gov (Y. Liu)

This is the author's manuscript of the article published in final edited form as:

Zhou, X., Li, T., Cui, Y., Meyerson, M., Weeks, J. A., Mullins, C. B., Jin, Y., Shin, H., Liu, Y., \& Zhu, L. (2020). Blade-Type Reaction Front in Micrometer-sized Germanium Particles during Lithiation. ACS Applied Materials \& Interfaces. https://doi.org/10.1021/acsami.0c13966 


\begin{abstract}
To investigate the lithium transport mechanism in micrometer-sized germanium (Ge) particles, in situ Focused Ion Beam-Scanning Electron Microscopy (FIB-SEM) was used to monitor the structural evolution of individual Ge particles during lithiation. Our results show that there are two types of reaction fronts during lithiation, representing the differences of reactions on the surface and in bulk. The cross-sectional SEM images and transmission electron microscopy characterizations show that the interface between amorphous $\mathrm{Li}_{\mathrm{x}} \mathrm{Ge}$ and $\mathrm{Ge}$ has a wedge shape due to the higher Li transport rate on the surface of the particle. The blade-type reaction front is formed at the interface of the amorphous $\mathrm{Li}_{\mathrm{x}} \mathrm{Ge}$ and crystalline $\mathrm{Ge}$, and is attributed to the large strain at the interface.
\end{abstract}

Keywords: Lithium ion battery, germanium, reaction front, lithiation, blade-type, in situ focused ion beam-scanning electron microscopy 


\section{Introduction}

Lithium ion batteries (LIBs) have become important energy storage technology for portable electronic devices and electric vehicles. ${ }^{1,2}$ There is a continual demand for new electrode materials with high capacity and good cycling stability. ${ }^{3,4}$ One promising direction is the use of alloying-type anode materials in LIBs such as Silicon (Si), Germanium (Ge) and Tin (Sn) because of their much higher capacity compared to graphite. ${ }^{5,6}$ However, the rapid capacity loss caused by the large volume change of such materials and the associated large stress induced structural damage upon cycling is a big hurdle regarding the employment of these materials in commercial applications. ${ }^{7}$ Since the volume change and stress are related to $\mathrm{Li}$ transport in the alloy particles during lithiation and delithiation, understanding Li transport behavior is critical to the design of active materials with high capacity and good cycling stability. In 2013, Wang, et. al discovered a two-phase process for electrochemical lithiation in amorphous Si by using in situ transmission electron microscopy (TEM). ${ }^{8}$ The lithiation occurred by the movement of a sharp phase boundary between the amorphous $\mathrm{Si}$ reactant and an amorphous $\mathrm{Li}_{x} \mathrm{Si}$ product. The twophase transport with a core-shell structure has also been discovered in nanometer-sized Ge, such as Ge nano-particles ${ }^{9}$ and Ge nano wires ${ }^{10}$. In recent years, computational studies using the twophase Li transport model with a core-shell structure have been conducted to understand $\mathrm{Li}$ transport behavior and stress/strain generation in $\mathrm{Si}$ and Ge particles with both micrometer and nanometer sizes during battery cycling. ${ }^{9,}{ }^{11-16}$ Although the two-phase core-shell model has been used in micrometer $(\mu \mathrm{m})$-sized alloy particles, there is not experimental evidence to validate it due to the sample size requirement for in situ TEM characterizatons. In addition to the core-shell structure with a sharp boundary, a finger-type Li transport process has been discovered in a twodimensional (2D) $\mathrm{NiO}$ nano-sheet during the lithiation process. ${ }^{17}$ It was interpreted that the finger-type reaction front is caused by the large tensile stress at the fingertip and the compressive stress around the finger. As the particle size increases, the Li diffusion-induced stress and strain also increase, which could affect the Li transport. It is clear that the two-phase core-shell model should be validated in $\mu \mathrm{m}$-sized particles. To investigate the behavior of $\mu \mathrm{m}$-sized electrode particles, we developed an in situ Focused Ion Beam-Scanning Electron Microscopy (FIB-SEM) method in our previous work. ${ }^{18,19}$ In this study, we modified the method and built an all solid- 
state single Ge particle battery inside the FIB-SEM to investigate the Li transport mechanism in $\mu \mathrm{m}$-sized Ge particles.

\section{Experimental Section}

The in situ experiment was performed on a Zeiss Nvision 40 FIB-SEM at the Center for Nanoscale Materials, Argonne National Laboratory. Figure S1 shows the schematic and SEM image of the experimental setup. Li metal was placed on the SEM stub as the negative electrode. A $\mu \mathrm{m}$-sized Ge particle was attached to a tungsten probe by FIB carbon deposition as the positive electrode. The Ge particles were prepared by jet milling as shown in our previous paper. ${ }^{20} \mathrm{~A}$ small piece of solid electrolyte $\mathrm{Li}_{6.4} \mathrm{La}_{3} \mathrm{Zr}_{1.4} \mathrm{Ta}_{0.6} \mathrm{O}_{12}$ (LLZTO) was placed on the $\mathrm{Li}$ metal. The preparation of LLTZO can be found in Ref. ${ }^{21}$. A Keithley 6430 sub-femtoamp remote sourcemeter was used to control the voltage to operate the single particle battery. During lithiation, the Ge particle was attached to the solid electrolyte and a bias voltage of $-0.01 \mathrm{~V}$ was applied to the Ge particle. The all solid single particle battery provides the opportunity to observe the particle morphology change in operando. SEM images were taken every $5 \mathrm{~s}$. To minimize the effect of the electron beam, we reduced the imaging current to about $40 \mathrm{pA}$, about $1 \%$ of the lithiation current. The TEM analysis was performed on a JEOL JEM2100F operated at $200 \mathrm{kV}$. The TEM sample was prepared by a standard FIB lift-out procedure.

\section{Results and Discussion}

Figure 1 shows the SEM images of a Ge particle at different states of lithiation. The lithiation process took about $5 \mathrm{~h}$. Figure 1a shows the Ge particle attached to the solid electrolyte before cycling. A bias voltage of $-0.01 \mathrm{~V}$ was applied to the particle. A sharp lithiation front appeared on the surface of the Ge particle shortly after the start of lithiation, as indicated by the red arrow in Figure 1b. It should also be noticed that before the lithiation started (Figure 1a), the left surface of the Ge particle is closely contacted to the solid electrolyte. After the lithiation started, the contact area reduced to a small point at the upper left corner. The non-uniform volume expansion can be attributed to different Li transport rates at the contact area. As the lithiation 
went on, the particle kept pushing the solid electrolyte and $\mathrm{W}$ probe away. These results indicate that electrode/electrolyte contact and volume expansion can be critical issues when Ge anode is used for the application of solid-state batteries. As the lithiation proceeded, the reaction front moved toward the $\mathrm{W}$ probe side, and a second reaction front appeared (Figure 1c - e, marked by yellow arrows). We noticed that the first reaction front was very clear and fast. There was almost no volume expansion coming along with it. The second reaction front was less distinct, but it brought volume expansion and cracks on the surface as indicated by green arrows. There was one large crack along the centerline and a few small cracks on the lower side of the particle. Video S1 shows the first 75 min of the lithiation process. The evolution of two reaction fronts are very clear in this video. The front of the large crack propagated simultaneously with the second reaction front. They reached the other side of the particle at about $75 \mathrm{~min}$ of lithiation.

Afterwards, the volume expansion became more homogenous and obvious (Figure $\mathrm{f}-\mathrm{k}$ ). Figure S2 shows the propagation of the two reaction fronts by measuring the distance between reaction fronts and the particle/electrolyte contact point during lithiation. The average speed of the first reaction front is about 2.3 times of the second reaction front. Video S2 shows the lithiation process from $75 \mathrm{~min}$ to the end. In the reported in situ TEM studies, the lithiation of Ge nanoparticles and nanowires was very fast on surface and followed the core-shell model. ${ }^{9,} 10$ In our experiment, the first reaction front represents the surface reaction, which took about $30 \mathrm{~min}$ to reach to the other side of the particle. The second reaction front along with volume expansion was due to the lithiation of bulk Ge, which took a longer time $(75 \mathrm{~min}$ ) to reach the other side. The results indicate that Li transport rate on the surface of $\mu \mathrm{m}$-sized Ge particle is still faster than the bulk, but not fast enough to form a core-shell structure as reported in nanoscale samples ${ }^{9,10}$. This can be attributed to the size difference. Also, the electron beam energy and current density employed in performing TEM are much higher than for SEM. ${ }^{22}$ High energy electrons may accelerate the surface reaction. Another Ge particle was tested under the same condition and had very similar performance. The lithiation process for the second Ge particle is shown in video S3. 

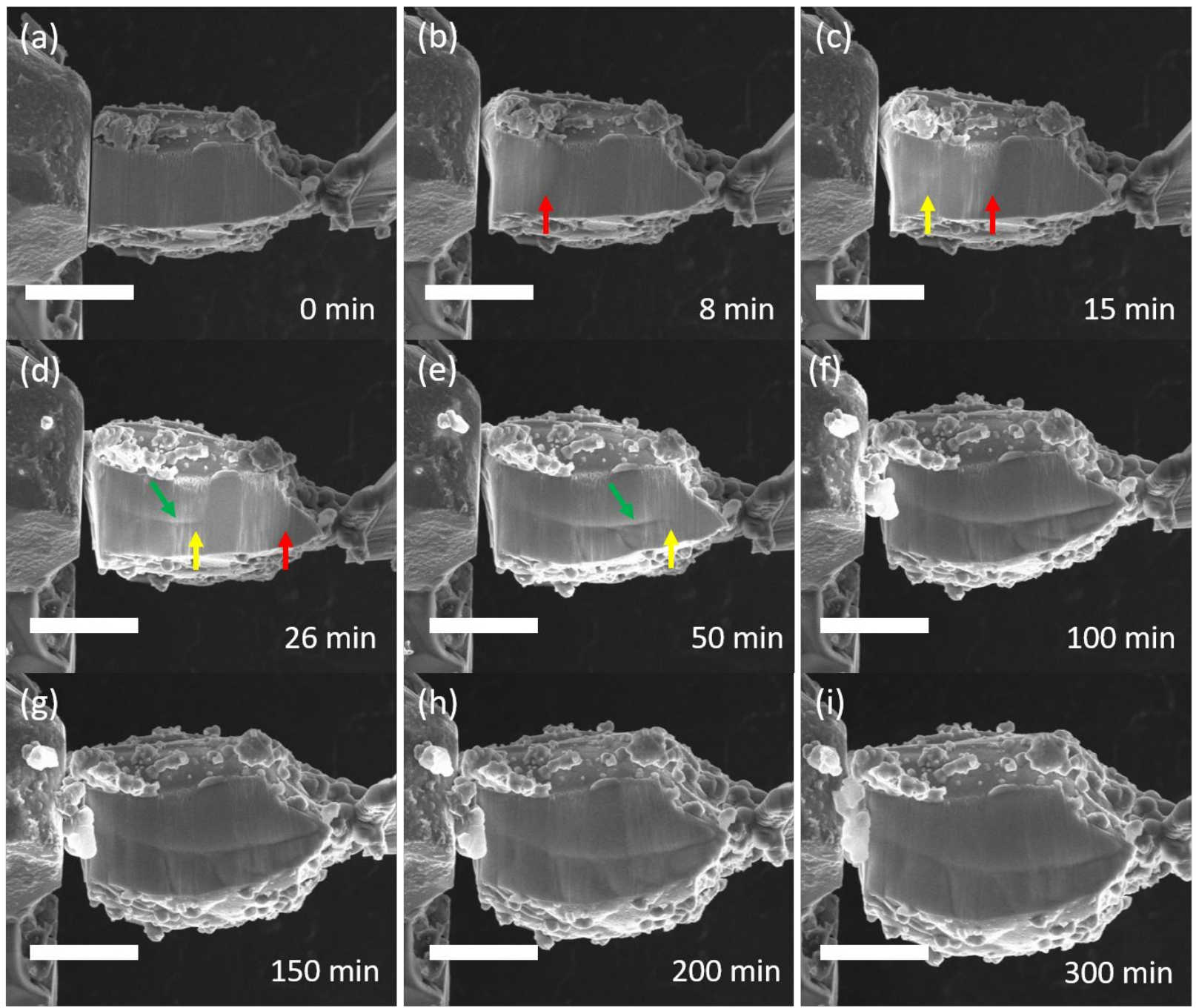

Figure 1. SEM images of morphology evolution of a germanium particle during lithiation. (a) Ge particle before lithiation. $(b-e)$ The evolution of reaction fronts. ( $f-i)$ Volume expansion after the second reaction front reached the other side. The scale bar is $5 \mu \mathrm{m}$.

To investigate the mechanism of reaction fronts, we partially lithiated a Ge particle. Figure 2a shows the SEM image of the pristine particle with a polished surface. The uniform contrast implies the single crystallinity of this particle. We stopped the lithiation when the second reaction front (bulk lithiation) reached across about $1 / 3$ of the particle width (Figure $2 b$ ). The lithiation process is shown in Video S4. Then we polished the Ge particle with FIB milling to check the lithiation conditions inside the particle. A SEM image was saved after each step of milling. There are clear interfaces between the lithiated amorphous $\mathrm{Li}_{\mathrm{x}} \mathrm{Ge}$ and unreacted crystal Ge in the cross-sectional images as shown in Figure 2. The reaction front has a wedge shape due 
to fast Li transport on the surface. Interestingly, the reaction front is not as smooth as expected. We also noticed there are spike-like reaction fronts in Figure 2c. Kai He et al. reported similar results in their study of lithiation of $2 \mathrm{D} \mathrm{NiO}$ nano-sheet electrodes. ${ }^{17}$ The particle was further milled by FIB to show the further insider (Figure 2d-f). Clearly, the spike-like reaction front is actually a blade-like reaction front in three dimensions (3D). As shown in Figure 2b, when the particle started to expand, the Ge particle was pushed away and the contact area is reduced to a small spot near the lower left corner. After further milling, close to the contact area as shown in Figure 2 d, we can observe that the blades spread out radially from the contact spot. It should be pointed out that the cracks (noted by green arrows) shown in Figure $2 \mathrm{~b}$ are correlated with the blades. It means the large strain introduced by the lithiation leads the crack indeed. In Figure 2c, some blades crossed with each other and resulted in isolated Ge areas. In Figure $2 \mathrm{e}$ and $\mathrm{f}$, the blades penetrated through unreacted Ge, reached the lithiated upper surface, leading to isolated Ge surrounded by $\mathrm{Li}_{\mathrm{x}} \mathrm{Ge}$. To prove the reproducibility of the results, anther Ge particle was tested at the same condition. The lithiation process and cross-sectional images are shown in Video S5 and Figure S3. The results of both particles are very similar. Our results clearly demonstrate that the core-shell reaction front was not observed. Instead, the blade-type reaction front is more likely. The formation of blade-type reaction fronts can be interpreted by the effect of stress/strain on lithium diffusivity in Ge particles. Large volume expansion during lithiation induces large strain at the interface between $\mathrm{Li}_{\mathrm{X}} \mathrm{Ge}$ and $\mathrm{Ge}$. The large strain could cause structure defects in unreacted Ge. In addition, intrinsic defects also exist in our $\mu \mathrm{m}$-sized Ge particles. Previous studies have shown that both intrinsic and strain-induced defects affect the local lithium diffusivity. ${ }^{23,24}$ In our study, the different lithium diffusivities due to defects at the reaction front initiated the formation of blades. As discussed in Ref ${ }^{17}$, the tensile stress concentration at the tip of the finger and the compressive stress around the finger result in spontaneous, long-aspect ratio growth of the finger in 2D NiO nano-sheet. In 3D $\mu \mathrm{m}$-sized Ge particles, the tip of blades are also under tensile stress concentration. Computational and experimental studies have shown that the tensile stress increases the lithium diffusivity while the compressive stress decrease it. ${ }^{25-28}$ Similar to the long-aspect ratio growth of the finger in $2 \mathrm{D} \mathrm{NiO}$ nano-sheet, in $\mu \mathrm{m}$-sized Ge particles, the tensile stress concentration at the tip of blade and the compressive stress around the blade resulted a long-aspect ratio growth. 

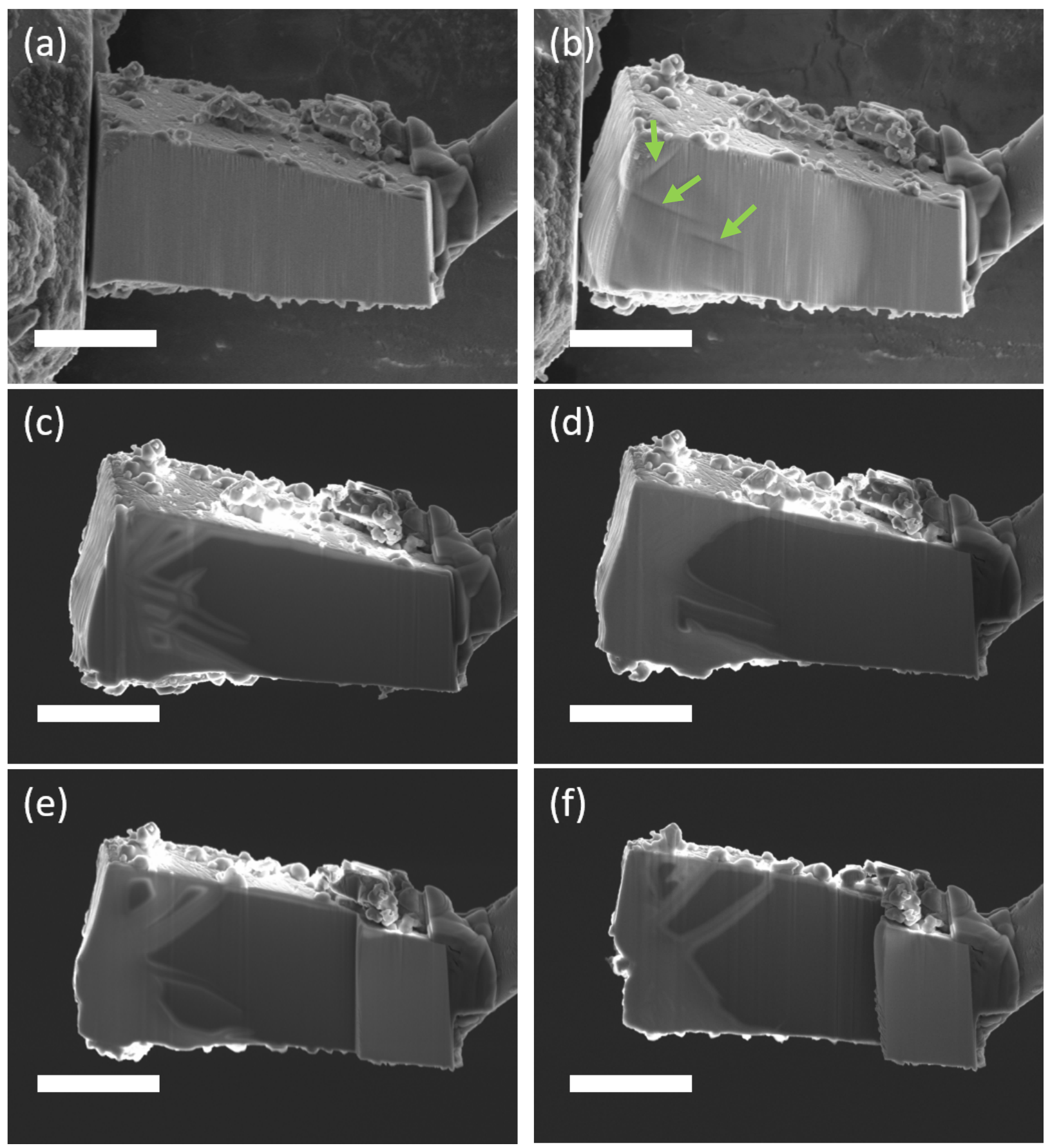

Figure 2. SEM images of a partially lithiated Ge particle. (a) Pristine Ge particle. (b) The same particle when lithiation was stopped. $(\mathrm{c}-\mathrm{f})$ Cross-sectional images at different states of FIB milling. The scale bar is $5 \mu \mathrm{m}$. 
To further investigate the reaction front, the second partially lithiated Ge particle was prepared into a TEM sample. Figure 3 shows the results of TEM characterizations. Figure $3 \mathrm{a}$ is a low magnification dark field TEM image. There is a sharp interface between area 1 (amorphous) and 2 (crystalline). Figure $3 \mathrm{~b}$ and $\mathrm{c}$ are the electron diffraction patterns of area 1 and 2 in Figure 3a, respectively. Area 1 is the lithiated amorphous $\mathrm{Li}_{\mathrm{x}} \mathrm{Ge}$, and area 2 is the unreacted crystalline Ge. The results confirm that the interface is indeed the reaction front. It is important to point out that the interface is curved. It means that the amorphous/crystal boundary does not exist in preferred crystal orientations. The reaction front does not propagate in preferred lattice planes. As shown in Figure 3a, the strips in area 2 (contrast variation) represent strains due to large volume expansion in the lithiated area. Figure $3 \mathrm{~d}$ is a high resolution TEM image (HREM) of the reaction front. The phase boundary between a- $\mathrm{Li}_{\mathrm{x}} \mathrm{Ge}$ and c-Ge is clearly shown in the image. We can conclude that the two-phase transport with a sharp boundary is still valid in $\mu \mathrm{m}$-sized Ge particles. A spike also appeared in the particle at the lower right corner in Figure 3a, which is likely a blade when viewed in 3D. Figure $3 \mathrm{e}$ and $\mathrm{f}$ are low magnification images of the beginning and the front end of the blade. As shown in Figure $3 \mathrm{e}, \mathrm{a}-\mathrm{Li}_{\mathrm{x}} \mathrm{Ge}$ just started to propagate into the blade. Figure S4a and $b$ are HREM and electron diffraction of the blade. The blade penetrated into unreacted Ge along the $<1 \overline{3} 1>$ facet in this case. The results indicate that it is still in the early stages of lithiation inside the spike, as most of the Ge retains its crystal structure. 

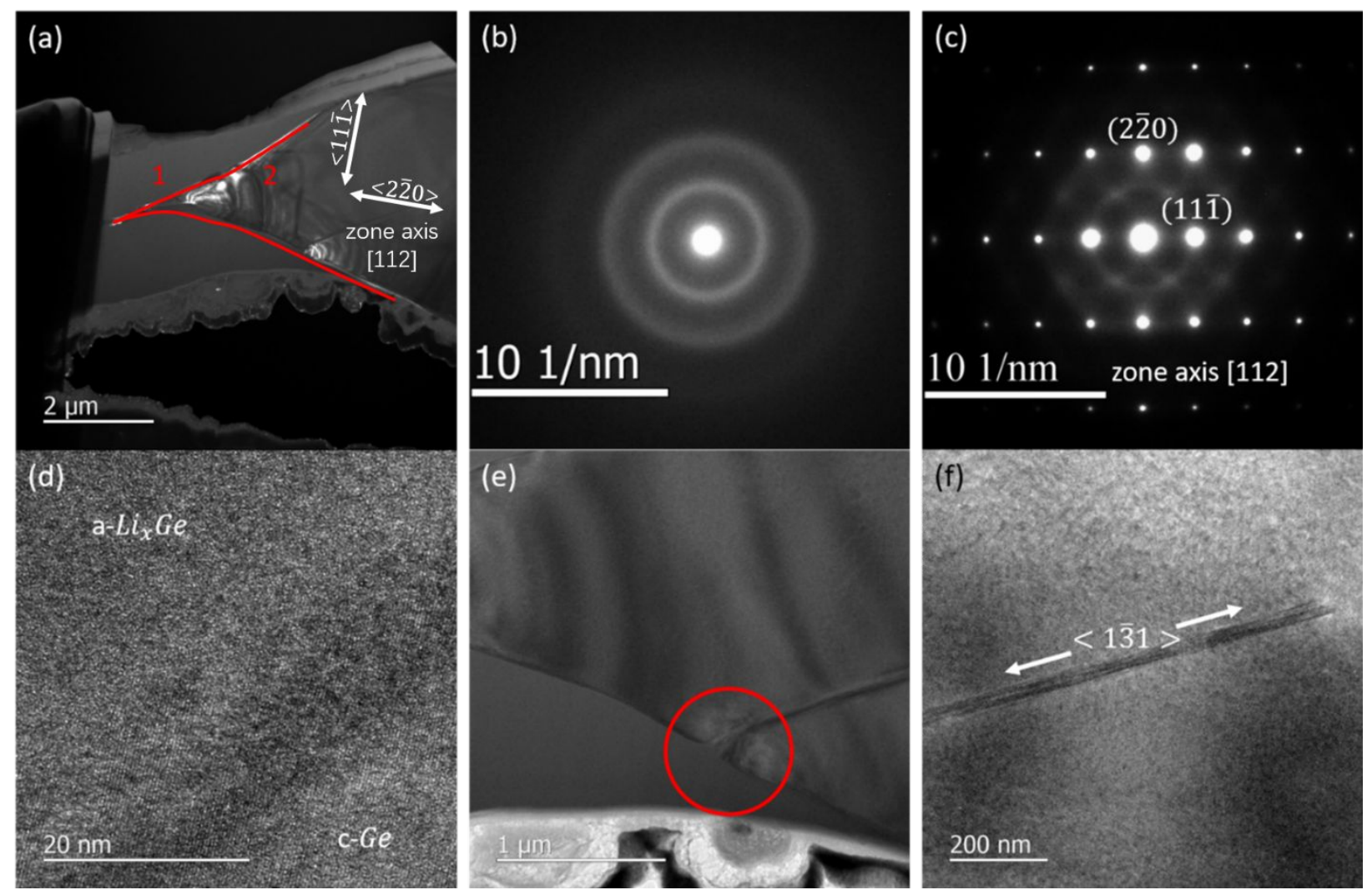

Figure 3. TEM images of a partially lithiated Ge particle. (a) A low magnification dark field image of the Ge particle. (b -c) Electron diffraction patterns of area 1 and 2 in Figure 3a. (d) A HREM image of the interface between area 1 and 2. (e-f) Low magnification dark field images of the spike.

\section{Conclusion}

In summary, we developed a solid-state in situ single particle battery in a FIB-SEM apparatus to study the dynamics of the propagation of the lithiation reaction front in $\mu \mathrm{m}$-sized Ge particles. The behavior of $\mu \mathrm{m}$-sized Ge particles is different from Ge nanoparticles/nanowires. In previous studies, the lithiation of Ge nanoparticles/nanowires follows a core-shell mode. While in our results, there are two reaction fronts in $\mu \mathrm{m}$-sized Ge during lithiation. The first reaction front represents a surface reaction and the second reaction front represents the lithiation of the bulk structure. The cross-sectional SEM images show the reaction front has a wedge shape due to fast $\mathrm{Li}$ transport on the surface. Moreover, blade-type structures are formed on the reaction front due to the large strain at the interface between $\mathrm{Li}_{\mathrm{X}} \mathrm{Ge}$ and Ge. Our results provide valuable insights into the lithiation mechanism of Ge, which can be used to design high capacity anode materials. 


\section{Acknowledgements}

This work was supported by the US National Science Foundation under Grant No.'s CBET1603847 (LZ) and CBET-1603491 (CBM). CBM also gratefully acknowledges support from the Robert A. Welch foundation through grant F-1436. This work was performed, in part, at the Center for Nanoscale Materials, an Office of Science user facility, was supported by the U.S. Department of Energy, Office of Science, Office of Basic Energy Sciences, under Contract No. DE-AC02-06CH11357.

Supporting Information Available: Schematic and SEM image of the experimental setup, additional SEM and TEM images of the blade-type reaction fronts, videos of lithiation processes of Ge particles.

\section{References}

1. Armand, M.; Tarascon, J. M., Building Better Batteries. Nature 2008, 451 (7179), 652657.

2. Nitta, N.; Wu, F.; Lee, J. T.; Yushin, G., Li-Ion Battery Materials: Present and Future. Materials Today 2015, 18 (5), 252-264.

3. Manthiram, A., Materials Challenges and Opportunities of Lithium Ion Batteries. The Journal of Physical Chemistry Letters 2011, 2 (3), 176-184.

4. Goodenough, J. B., Electrochemical Energy Storage in A Sustainable Modern Society. Energy \& Environmental Science 2014, 7 (1), 14-18.

5. Nitta, N.; Yushin, G., High-Capacity Anode Materials for Lithium-Ion Batteries: Choice of Elements and Structures for Active Particles. Particle \& Particle Systems Characterization 2014, 31 (3), 317-336.

6. Tian, H.; Xin, F.; Wang, X.; He, W.; Han, W., High Capacity Group-IV Elements (Si, Ge, Sn) Based Anodes for Lithium-Ion Batteries. Journal of Materiomics 2015, 1 (3), 153-169. 7. Liu, Y.; Zhang, S.; Zhu, T., Germanium-Based Electrode Materials for Lithium-Ion Batteries. ChemElectroChem 2014, 1 (4), 706-713.

8. Wang, J. W.; He, Y.; Fan, F.; Liu, X. H.; Xia, S.; Liu, Y.; Harris, C. T.; Li, H.; Huang, J. Y.; Mao, S. X.; Zhu, T., Two-Phase Electrochemical Lithiation in Amorphous Silicon. Nano Letters 2013, 13 (2), 709-715. 
9. Liang, W.; Yang, H.; Fan, F.; Liu, Y.; Liu, X. H.; Huang, J. Y.; Zhu, T.; Zhang, S., Tough Germanium Nanoparticles under Electrochemical Cycling. ACS Nano 2013, 7 (4), $3427-$ 3433.

10. Liu, X. H.; Huang, S.; Picraux, S. T.; Li, J.; Zhu, T.; Huang, J. Y., Reversible Nanopore Formation in Ge Nanowires during Lithiation-Delithiation Cycling: An In Situ Transmission Electron Microscopy Study. Nano Letters 2011, 11 (9), 3991-3997.

11. Wang, X.; Wang, B.; Meyerson, M.; Mullins, C. B.; Fu, Y.; Zhu, L.; Chen, L., A Phase-Field Model Integrating Reaction-Diffusion Kinetics and Elasto-Plastic Deformation with Application to Lithiated Selenium-Doped Germanium Electrodes. International Journal of Mechanical Sciences 2018, 144, 158-171.

12. Wang, X.; Yenusah, C. O.; Tantratian, K.; Meyerson, M. L.; Guo, A.; Mullins, C. B.; Zhu, L.; Chen, L., Understanding the Mechanism of Stress Mitigation in Selenium-Doped Germanium Electrodes. Journal of The Electrochemical Society 2019, 166 (2), A364-A377. 13. Liu, X. H.; Zhong, L.; Huang, S.; Mao, S. X.; Zhu, T.; Huang, J. Y., Size-Dependent Fracture of Silicon Nanoparticles During Lithiation. ACS Nano 2012, 6 (2), 1522-1531.

14. Liu, X. H.; Zheng, H.; Zhong, L.; Huang, S.; Karki, K.; Zhang, L. Q.; Liu, Y.; Kushima, A.; Liang, W. T.; Wang, J. W.; Cho, J.-H.; Epstein, E.; Dayeh, S. A.; Picraux, S. T.; Zhu, T.; Li, J.; Sullivan, J. P.; Cumings, J.; Wang, C.; Mao, S. X.; Ye, Z. Z.; Zhang, S.; Huang, J. Y., Anisotropic Swelling and Fracture of Silicon Nanowires during Lithiation. Nano Letters 2011, 11 (8), 3312-3318.

15. Huang, S.; Fan, F.; Li, J.; Zhang, S.; Zhu, T., Stress Generation during Lithiation of High-Capacity Electrode Particles in Lithium Ion Batteries. Acta Materialia 2013, 61 (12), 43544364.

16. Zhang, X.; Krischok, A.; Linder, C., A Variational Framework to Model Diffusion Induced Large Plastic Deformation and Phase Field Fracture during Initial Two-Phase Lithiation of Silicon Electrodes. Computer Methods in Applied Mechanics and Engineering 2016, 312, 5177.

17. He, K.; Xin, H. L.; Zhao, K.; Yu, X.; Nordlund, D.; Weng, T.-C.; Li, J.; Jiang, Y.; Cadigan, C. A.; Richards, R. M.; Doeff, M. M.; Yang, X.-Q.; Stach, E. A.; Li, J.; Lin, F.; Su, D., Transitions from Near-Surface to Interior Redox upon Lithiation in Conversion Electrode Materials. Nano Letters 2015, 15 (2), 1437-1444.

18. Zhou, X.; Li, T.; Cui, Y.; Fu, Y.; Liu, Y.; Zhu, L., In Situ Focused Ion Beam Scanning Electron Microscope Study of Microstructural Evolution of Single Tin Particle Anode for Li-Ion Batteries. ACS Applied Materials \& Interfaces 2019, 11 (2), 1733-1738.

19. Zhou, X.; Li, T.; Cui, Y.; Meyerson, M. L.; Mullins, C. B.; Liu, Y.; Zhu, L., In Situ Focused Ion Beam-Scanning Electron Microscope Study of Crack and Nanopore Formation in Germanium Particle During (De)lithiation. ACS Applied Energy Materials 2019, 2 (4), 2441 2446.

20. Klavetter, K. C.; Pedro de Souza, J.; Heller, A.; Mullins, C. B., High Tap Density Microparticles of Selenium-Doped Germanium as A High Efficiency, Stable Cycling LithiumIon Battery Anode Material. Journal of Materials Chemistry A 2015, 3 (11), 5829-5834.

21. Jin, Y.; Liu, K.; Lang, J.; Jiang, X.; Zheng, Z.; Su, Q.; Huang, Z.; Long, Y.; Wang, C.-a.; Wu, H.; Cui, Y., High-Energy-Density Solid-Electrolyte-Based Liquid Li-S and Li-Se Batteries. Joule 2020, 4 (1), 262-274.

22. Egerton, R. F.; Li, P.; Malac, M., Radiation Damage in The TEM and SEM. Micron 2004, 35 (6), 399-409. 
23. Lin, Y.-C.; Kim, D.; Li, Z.; Nguyen, B.-M.; Li, N.; Zhang, S.; Yoo, J., Strain-Induced Structural Defects and Their Effects on The Electrochemical Performances of Silicon Core/Germanium Shell Nanowire Heterostructures. Nanoscale 2017, 9 (3), 1213-1220.

24. Bordes, A.; De Vito, E.; Haon, C.; Boulineau, A.; Montani, A.; Marcus, P., Multiscale Investigation of Silicon Anode Li Insertion Mechanisms by Time-of-Flight Secondary Ion Mass Spectrometer Imaging Performed on an In Situ Focused Ion Beam Cross Section. Chemistry of Materials 2016, 28 (5), 1566-1573.

25. Ding, B.; Wu, H.; Xu, Z.; Li, X.; Gao, H., Stress Effects on Lithiation in Silicon. Nano Energy 2017, 38, 486-493.

26. McDowell, M. T.; Ryu, I.; Lee, S. W.; Wang, C.; Nix, W. D.; Cui, Y., Studying the Kinetics of Crystalline Silicon Nanoparticle Lithiation with In Situ Transmission Electron Microscopy. Advanced Materials 2012, 24 (45), 6034-6041.

27. Liu, X. H.; Fan, F.; Yang, H.; Zhang, S.; Huang, J. Y.; Zhu, T., Self-Limiting Lithiation in Silicon Nanowires. ACS Nano 2013, 7 (2), 1495-1503.

28. Zhao, K.; Pharr, M.; Wan, Q.; Wang, W. L.; Kaxiras, E.; Vlassak, J. J.; Suo, Z., Concurrent Reaction and Plasticity during Initial Lithiation of Crystalline Silicon in Lithium-Ion Batteries. Journal of The Electrochemical Society 2012, 159 (3), A238-A243. 


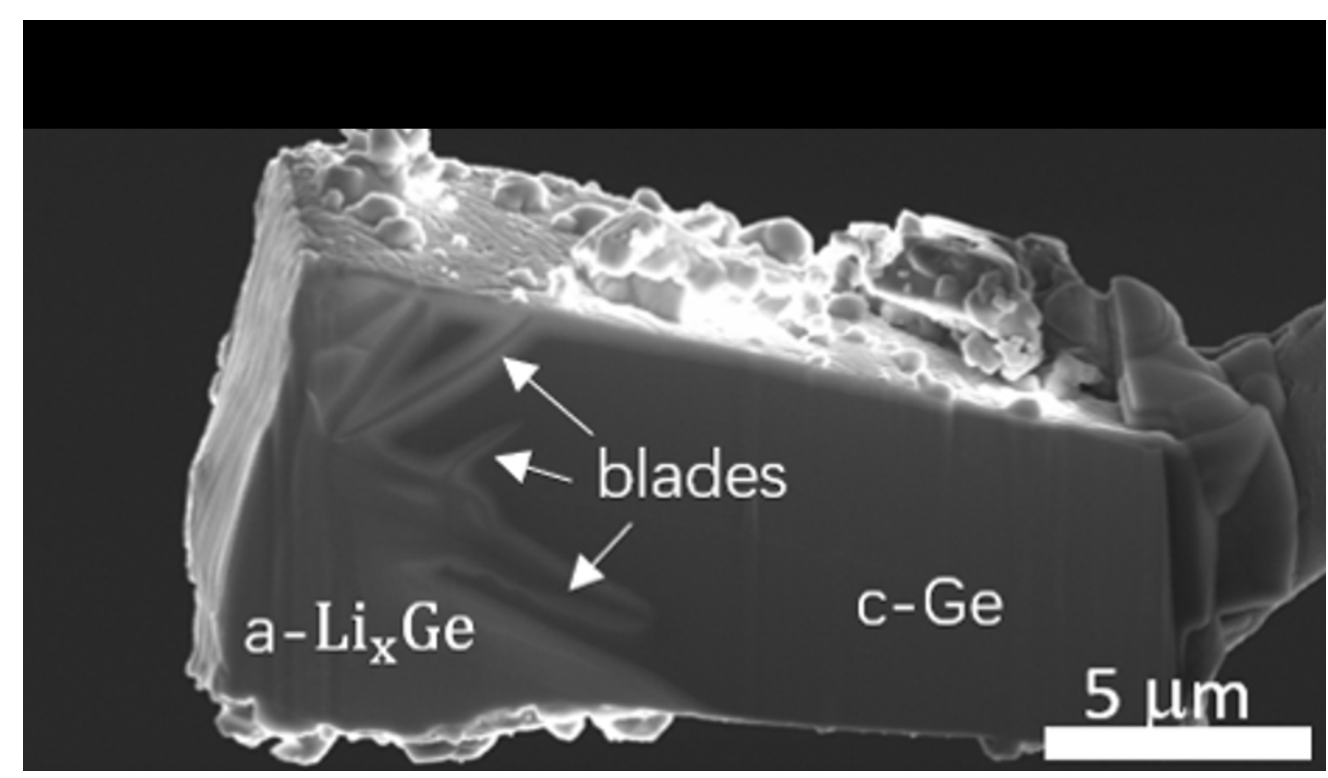

$170 \times 98 \mathrm{~mm}(150 \times 150 \mathrm{DPI})$ 